\title{
Mammary Duct Ectasia
}

National Cancer Institute

\section{Source}

National Cancer Institute. Mammary Duct Ectasia. NCI Thesaurus. Code C3522.

Dilatation of the lumen of the mammary ducts without evidence of epithelial atypia. 